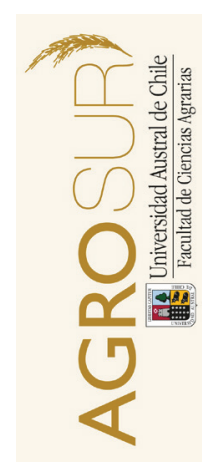

\title{
Variación espacial de propiedades físicas de un suelo derivado de cenizas volcánicas sometido a distintas estrategias de mejoramiento de una pradera degradada
}

\author{
Spatial variation of physical properties of a soil derived from volcanic ash \\ subjected to different strategies of improvement of a degraded pasture

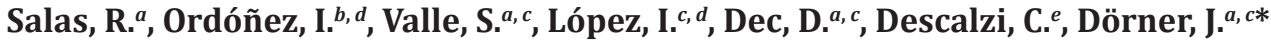 \\ ${ }^{a}$ Instituto de Ingeniería Agraria y Suelos, Universidad Austral de Chile. Casilla 567, Valdivia, Chile. \\ ${ }^{b}$ Magister en Ciencias Mención Producción Animal, Escuela de Graduados, Facultad de Ciencias Agrarias, \\ Universidad Austral de Chile. \\ ${ }^{c}$ Centro de Investigación en Suelos Volcánicas, Universidad Austral de Chile.
}

${ }^{d}$ Institute of Agriculture and Environment, Massey University, Private Bag 11222, Palmerston North, New Zealand.

${ }^{e}$ Programa de Doctorado en Ciencias Agrarias, Escuela de Graduados, Facultad de Ciencias Agrarias, Universidad Austral de Chile.

\begin{tabular}{l} 
A R T I C L E I N F O \\
\hline Article history: \\
Received 24.11.2016 \\
Accepted 19.01.2017 \\
\hline Keywords: \\
Soil physical quality \\
Grazing \\
Pasture improvement \\
\hline Original Research Article, \\
Special Issue: Pastures for \\
Sustainable Productions Systems \\
\hline *Corresponding author: \\
Rosemarie Salas, José Dörner \\
E-mail address: \\
josedorner@uach.cl
\end{tabular}

\section{A B S T R A C T}

Southern Chile presents adequate conditions to develop sheepherding ecosystems. However, from the total of pastures surface in both regions (Los Ríos and Los Lagos) approximately there is a $44 \%$ of degraded pastures. Thus, this work is aimed to assess the effects of pastures improvement strategies on spatial variability of soil physical quality. The experiment was realized in the Estación Experimental Agropecuaria Austral, with five pastures on an Andisol: NsF) Naturalized pasture without fertilization; NcF) Improved naturalized pasture with fertilization; Lp+Tr ST) sown Lolium perenne L., Trifolium repens L. pasture with traditional tillage; Lp+Tr CL) sown L.perenne, Trepens pasture with cero tillage; CL) sown pasture with several species L. perenne, T. repens, Bromus valdivianus Phil, Dactylis glomerata L. and Holcus lanatus L. under cero tillage. Following a grid the following parameters were determined: volumetric water content $(\theta \mathrm{c})$, air conductivity $(\mathrm{Kl})$ and penetration resistance (PR). The data collection was on November 2, 2015, where moderate spatial dependence was determined for all parameters, such that sectors with higher $\theta \mathrm{c}(20 \mathrm{Vol} . \%)$ in the soil were those that presented lower RP $(2,122 \mathrm{kPa})$ and $\mathrm{Kl}\left(0.83 \mathrm{~cm} \mathrm{~s}^{-1}\right)$. The 30 months since the establishment of pasture improvement strategies have allowed a stabilization of the soil structure that is reflected in the low impact of sheep grazing on the physical parameters evaluated.

\section{RESUMEN}

La zona Sur de Chile presenta condiciones edafoclimáticas adecuadas para el desarrollo de ecosistemas pastoriles. Sin embargo, del total de praderas existentes en las regiones de Los Ríos y Los Lagos, aproximadamente el 44\% aún son de baja producción (menor de $4.000 \mathrm{~kg} \mathrm{MS} \mathrm{ha}^{-1} \mathrm{año}^{-1}$ ). El objetivo de este trabajo fue evaluar los efectos de estrategias de mejoramiento de praderas sobre la variación espacial de la calidad física del suelo. El ensayo se realizó en la Estación Experimental Agropecuaria Austral, en cinco praderas sobre un Andisol: NsF) Pradera natural sin fertilizar; NcF) Pradera natural fertilizada; Lp+Tr ST) Pradera de Lolium perenne L., Trifolium repens L. con siembra tradicional; Lp+Tr CL) Pradera de L. perenne, T. repens con cero labranza; Polifítica CL) Pradera polifitica de L. perenne, Trepens, Bromus valdivianus Phil, Dactylis glomerata L. y Holcus lanatus L. con cero labranza. En una grilla $(5 \mathrm{x} 5 \mathrm{~m})$ se determinó el contenido volumétrico de agua $(\theta \mathrm{c})$, la conductividad de aire $(\mathrm{Kl})$ y la resistencia a la penetración (RP) del suelo. La recolección de datos fue el 2 de noviembre del 2015 donde se determinó una moderada dependencia espacial para todos los parámetros evaluados, tal que sectores con mayor $\theta$ c (20 Vol. \%) en el suelo fueron los que presentaron menor RP (2.122 kPa) y Kl $\left(0,83 \mathrm{~cm} \mathrm{~s}^{-1}\right)$. Los 30 meses desde el establecimiento de las estrategias de mejoramiento de praderas han permitido una estabilización de la estructura del suelo que se refleja en el bajo impacto del pastoreo ovino sobre los parámetros físicos.

Palabras clave: calidad física de suelos, pastoreo, mejoramiento de praderas. 


\section{INTRODUCCIÓN}

Las praderas son el principal recurso forrajero para los animales rumiantes de producción en el sur de Chile (Balocchi, 2002). De la superficie de praderas en la región de Los Ríos y Los Lagos el 91,3\% corresponde a praderas naturalizadas y mejoradas y sólo un $8,7 \%$ son artificiales (INE, 2007). La pradera natural es aquella con una comunidad polifítica que se ha establecido espontáneamente en condición pobre, siendo de bajo rendimiento anual (menor de $4.000 \mathrm{~kg} \mathrm{MS} \mathrm{ha}^{-1} \mathrm{año}^{-1}$ ). En ese contexto, para Siebald (2001) el mejoramiento de praderas naturalizadas es una alternativa de bajo requerimiento de capital, que aumenta significativamente la producción y calidad de forraje, a través de tres métodos: i) fertilización y manejo de pastoreo, ii) regeneración de praderas y iii) establecimiento de praderas (Cuevas 1980; Balocchi y López, 1994).

La fertilización y manejo de pastoreo consiste en mejorar los niveles de nutrientes y condiciones químicas del suelo, además de integrar un criterio de pastoreo de entrada y salida del rebaño, incluyendo cortes de limpieza que incrementan la participación de especies de alto valor forrajero (Cuevas, 1980; Balocchi y López, 1994). La regeneración de praderas consiste en integrar al suelo semillas de alto valor forrajero y fertilizante en una hilera, a fin de aumentar el rendimiento de forma rápida y sin alterar la comunidad vegetal residente. El establecimiento de praderas se logra con una labranza tradicional del suelo para, posteriormente, sembrar especies de alto valor forrajero y aumentar la producción (Torres, 2006).

Dörner et al. (2012) señalan que luego del uso de la labranza tradicional propiedades físicas de un Andisol como la porosidad total, conductividad hidráulica saturada y permeabilidad aumentan, lo que está asociado a una disminución de la densidad aparente y de la resistencia mecánica en comparación a cero labranza o a la situación inicial del suelo antes de ser intervenido. Sin embargo, esta situación cambia en el corto plazo debido al reordenamiento de las partículas y formación/destrucción de estructura, p. ej. luego de eventos de mojado y secado y presiones externas como el pastoreo. Por lo tanto, el espacio poroso entre agregados presenta un comportamiento dinámico, ya que la estructura se forma, se desintegra y se reorganiza de manera periódica (Horn y Baumgartl, 2001). Esto no deja de ser relevante ya que indica que el volumen de poros, que regula la relación agua/aire, dista de ser constante y, al contrario, es dinámico y variable en el tiempo debido a los ciclos de mojado y secado (Dec et al., 2011), al manejo del cultivo (Dörner et al., 2012) y eventos de pastoreo (Dec et al., 2012). Además, se debe considerar que el suelo, tiene la capacidad de recuperarse, siempre que las alteraciones antrópicas no sean muy drásticas y tenga el tiempo suficiente para que los procesos de mantención y recuperación (resiliencia) ocurran (Dörner et al., 2011; Dörner et al., 2013).

El comportamiento dinámico de la estructura del suelo influye sobre la variabilidad de sus propiedades, ya sea en el espacio y el tiempo (Jaramillo, 2008; Nielsen y Wendroth, 2003). La variabilidad de las propiedades del suelo en el espacio, pueden provenir de características inherentes a procesos de formación de suelo o bien producto de acciones antrópicas. Es decir, la historia, el clima, la topografía, la actividad biológica y la acción humana (Nielsen y Wendroth, 2003).

Se han llevado a cabo estudios de parámetros de calidad física en suelos derivados de materiales volcánicos bajo pastoreo tomando en cuenta la variabilidad espacial por (Dec et al., 2011) y temporal por (Dec et al., 2012; Zúñiga et al., 2015; Ivelic-Sáez et al., 2015) que demuestran que estos presentan excelentes propiedades físicas. Sin embargo, no existe mayor información del impacto que puede tener la implementación de distintas estrategias de mejoramiento de praderas bajo pastoreo sobre la variabilidad espacial de propiedades que dependen de la estructura del suelo. Lo anterior es relevante ya que permite conocer la respuesta del suelo frente al pastoreo, luego de haber sido expuesto a distintas medidas de manejo para mejorar la productividad de la pradera. En ese contexto, el objetivo del trabajo es evaluar el efecto de distintas estrategias de mejoramiento de una pradera degradada, que se encuentra bajo condiciones de pastoreo ovino, sobre la variación espacial del contenido volumétrico de agua, resistencia a la penetración y conductividad de aire del suelo, en condiciones de campo.

\section{MATERIAL Y MÉTODOS}

\section{Caracterización edafo-climática del lugar de ensayo}

El ensayo se ubicó en la Estación Experimental Agropecuaria Austral (EEAA), perteneciente a la Universidad Austral de Chile, que se encuentra en la Región de los Ríos, ciudad de Valdivia. Se ubica geográficamente entre los paralelos $39^{\circ} 45^{\prime} 30^{\prime \prime}$ a $39^{\circ} 47^{\prime} 30^{\prime \prime}$ latitud sur y los meridianos $73^{\circ} 14^{\prime} 55^{\prime \prime}$ y $73^{\circ} 13^{\prime} 5^{\prime \prime}$ longitud oeste.

El clima templado lluvioso con influencia mediterránea con temperaturas medias anuales de $12{ }^{\circ} \mathrm{C}$, precipitaciones promedio de $2500 \mathrm{~mm}$ distribuidas estacionalmente y vientos predominantes provenientes del Norte y el Oeste (Huber, 1970).

El suelo del ensayo correspondió a un Andisol (localmente denominado Trumao), de la serie Valdivia, miembro de la familia media mésica de los Duric Hapludands (CIREN, 2003). El suelo es moderadamente profundo, ocupa una posición de terrazas remanentes en la depresión de San José, a una altura de 9 a 
20 m.s.n.m. Además, presenta una textura superficial franco limoso y color pardo oscuro en el matiz 7.5 YR y textura franco arenosa y color pardo oscuro en el matiz 7.5 YR en profundidad. El substrato corresponde a una toba, depósito de materiales volcánicos de fuerte compactación y mezclado con clastos alterados, el cual se denomina localmente Cancagua. De topografía plana, con una profundidad mayor a dos metros, y con una capacidad de uso potencial clase II. Mayores detalles respecto al suelo bajo distintos tipos de praderas y usos de suelo se pueden encontrar en Zúñiga et al. (2015) y Dörner et al. (2015).

\section{Estrategias de mejoramiento en praderas}

El estudio se compuso de 4 tratamientos, más una pradera degradada que constituye el control (Cuadro 1), con un diseño experimental de bloques completos al azar (3 bloques), dando un total de 15 parcelas con una superficie de $400 \mathrm{~m}^{2}$ cada una ( $20 \mathrm{~m} \times 20 \mathrm{~m}$ ). Se compuso de cuatro tratamientos, más una pradera degradada que constituye el control. En marzo de 2013 se delimitaron las 15 parcelas a evaluar y en abril del mismo año se realizó la siembra.

Los tratamientos fueron los siguientes: pradera natural sin fertilización (NsF) que correspondió a la condición inicial; pradera natural con fertilización $(\mathrm{NcF})$ pradera inicial, pero con corrección de nutrientes de acuerdo a las condiciones químicas del suelo; pradera de Lolium perenne L. y Trifolium repens L. establecida con labranza tradicional(Lp+Tr ST), pradera mixta de $L$. perenne con T. repens con cero labranza, sembrada con regeneradora ( $\mathrm{Lp}+\mathrm{Tr} \mathrm{CL}$ ), con una dosis de semilla utilizada de 25,2 $\mathrm{kg} \mathrm{ha}^{-1}$ para Lolium perenne L. y 3,9 $\mathrm{kg} \mathrm{ha}^{-1}$ para Trifolium repens L. en ambos casos, y por último, pradera polifítica con cero labranza (Polifítica $\mathrm{CL})$, la cual corresponde a una pradera con L. perenne (dosis de semilla 6,3 $\mathrm{kg} \mathrm{ha}^{-1}$ ), Bromus valdivianus Phil. (dosis de $17 \mathrm{~kg} \mathrm{ha}^{-1}$ ), Dactylis glomerata L. (dosis de $1 \mathrm{~kg}$ ha $^{-1}$ )., Holcus lanatus L. (dosis de $1 \mathrm{~kg} \mathrm{ha}^{-1}$ ). y T. repens (dosis de 3,9 $\mathrm{kg} \mathrm{ha}^{-1}$ ) que se sembró con regeneradora. Para Lp+Tr CL y Polifítica CL, se realizó un barbecho químico con glifosato, la dosis aplicada fue de $3 \mathrm{~L} \mathrm{ha}^{-1}$. Posteriormente, en abril del 2013 se realizó la siembra directa mediante una regeneradora de marca Semeato, que tiene una profundidad de siembra de $1,5 \mathrm{~cm}$, distancia entre hilera de $17 \mathrm{~cm}$ y un ancho de trabajo de 3,4 metros.

Respecto al establecimiento de Lp+Tr ST, se compuso de: una aplicación de glifosato (3 L ha-1) para eliminar la pradera residente, el uso de arado de disco (una vez), rastra (dos veces) y vibrocultivador, este último se utilizó para eliminar toda estructura remanente del suelo y emparejar el sitio a sembrar. Posteriormente, en abril del 2013, se sembró en hileras, mediante una sembradora Baldan, a una profundidad de $1,5 \mathrm{~cm}$, la distancia entre hileras es de $17 \mathrm{~cm}$ y un ancho de trabajo de 2,7 metros. La labor se finalizó con el paso de un rodillo compactador, que permite un mejor contacto de la semilla con el suelo.

Todas las parcelas se fertilizaron con la misma cantidad de nutrientes, independiente del tratamiento. Además, se aplicó una enmienda de carbonato de calcio, con una dosis de $2.000 \mathrm{~kg} \mathrm{ha}^{-1}$. Posteriormente se realizaron aplicaciones anuales de fertilizante en forma de cobertera.

\section{Recolección de datos}

Se recolectaron datos in situ de conductividad de aire $(\mathrm{Kl})$, contenido volumétrico de agua del suelo $\left(\theta_{c}\right)$ y resistencia a la penetración (RP) del suelo en retículo regular con una distancia de 5 metros, en ambas direcciones (Figura 1), el largo y ancho total del ensayo corresponde a $100 \mathrm{~m}$ y $60 \mathrm{~m}$ respectivamente dando un área total estudio de $6.000 \mathrm{~m}^{2}$. El muestreo se rea-

Cuadro 1. Manejo del suelo y composición botánica de las estrategias de mejoramiento en praderas.

Table 1. Soil management and botanical composition of pasture improvement strategies.

\begin{tabular}{|c|c|c|}
\hline Tratamiento & Tipo de labranza & Composición botánica \\
\hline NsF & Sin labranza & $\begin{array}{l}\text { Lolium perenne L., Holcus Lanatus L., Trifolium repens L., } \\
\text { otras gramíneas (de bajo valor forrajero) y Hoja ancha (testigo) }\end{array}$ \\
\hline $\mathrm{NcF}$ & Sin labranza & $\begin{array}{l}\text { Lolium perenne L., Holcus lanatus L., Trifolium repens L., } \\
\text { otras gramíneas (de bajo valor forrajero) y Hoja ancha }\end{array}$ \\
\hline Lp+Tr ST & Labranza tradicional & Lolium perenne L. y Trifolium repens L. \\
\hline$L p+\operatorname{Tr} C L$ & Cero labranza & Lolium perenne L. y Trifolium repens L. \\
\hline Polifítica CL & Cero labranza & $\begin{array}{c}\text { Lolium perenne L., Trifolium repens L., Bromus valdivianus Phil., } \\
\text { Dactylis glomerata L. y Holcus Lanatus L. }\end{array}$ \\
\hline
\end{tabular}




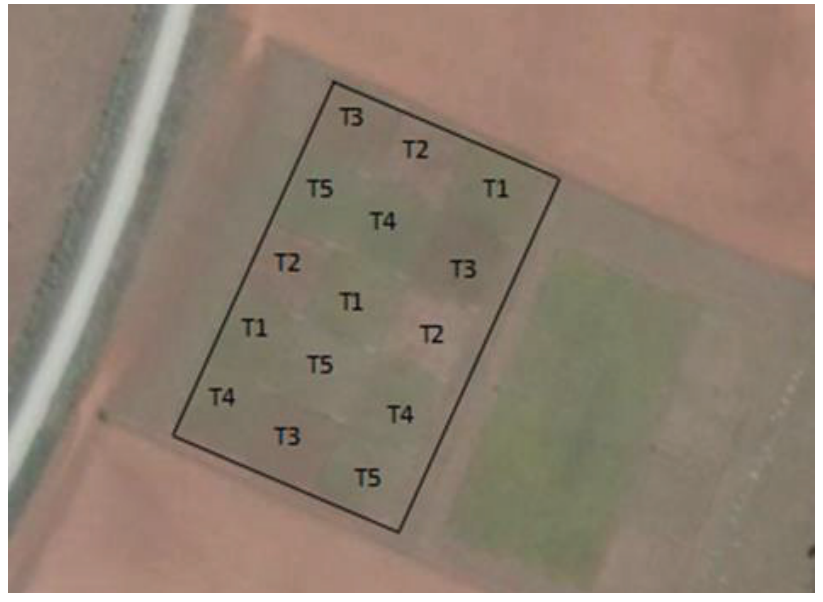

Figura 1. Distribución de estrategias de mejoramiento en campo.

Figure 1. Distribution of the pasture improvement strategies on the field.

lizó el 2 noviembre 2015, antes y después de un evento de pastoreo, en donde la disponibilidad de entrada para los animales fue de $1.700 \mathrm{~kg} \mathrm{MS} \mathrm{ha-1}$. Las praderas fueron pastoreadas con 25 ovejas por parcela, lo que corresponde a una carga animal instantánea de 625 ovejas por ha-1 ${ }^{-1}$ Estos parámetros fueron medidos en la superficie del suelo (Kl), en los primeros $10 \mathrm{~cm}$ $\left(\theta_{c}\right.$ y RP).

Por cada tratamiento se recolectaron 27 datos para conductividad de aire, contenido volumétrico de agua y resistencia a la penetración del suelo, dando un $\mathrm{N}$ total de 135 para cada parámetro evaluado.

Primero se midió la conductividad de aire a nivel superficial del suelo, a través de un permeámetro de aire (Air permeability PL-300), instrumento que mide la velocidad con que fluye el aire del suelo, a través del sistema poroso. Su funcionamiento se basa en una cámara de medición que se coloca sobre la superficie del suelo, en donde previamente se ha eliminado la cobertura vegetal, definiendo el área de medición para el flujo del aire. Para determinar la velocidad con que fluye el aire, se generó una diferencia de presión en la cámara que permite un flujo de aire por convección, el que es calculado con la ecuación de Darcy (Eijkelkamp, 2014).

Posteriormente, y en el mismo lugar, se determinó el contenido volumétrico de agua del suelo con un WET-2-KI (Delta-T Devices Ltd, England). Finalmente, se registró la resistencia a la penetración del suelo con un penetrómetro (Hand Penetrometer, Eijekelkamp Agrisearch Equipment, Giesbeek, The Netherlands) que permite medir la resistencia que ejerce el suelo a ser penetrado, es decir, la resistencia que tiene el suelo a ser deformado por algún estrés mecánico.

\section{Análisis estadísticos}

Para la descripción estadística de los datos espaciales de contenido volumétrico de agua, resistencia a la penetración y conductividad de aire del suelo, se calcularon los siguientes parámetros: media, mediana, valores máximos y mínimos, además de coeficiente de variación, skewness y kurtosis. Para determinar diferencias significativas por efecto de pastoreo en cada una de las estrategias se realizó un ANDEVA, posterior a esto se hizo un test de Tukey con un 95\% de confianza $(\mathrm{p}<0,05)$

La estructura de la variabilidad espacial fue analizada usando el software geoestadístico GS + versión 9.0. Este programa permite la definición de semivariogramas y sus respectivos sets de parámetros (nugget Co; sill Co + C; range Ao). El mejor modelo matemático fue elegido basándose en el más alto coeficiente de determinación $\left(\mathrm{R}^{2}\right)$ y en la más baja suma residual (RSS). El grado de dependencia (DD) fue calculado usando la razón Co / $(\mathrm{Co}+\mathrm{C})$, propuesta por Cambardella et al. (1994).

\section{RESULTADOS}

\section{Variabilidad espacial de algunas propiedades físicas del suelo bajo distintas estrategias de mejoramiento en una pradera degradada}

Los resultados del análisis estadístico de las estrategias de mejoramiento evaluadas no mostraron diferencias estadísticas $(p>0,05)$ entre tratamientos para el contenido volumétrico de agua en el suelo (Cuadro 2). Sin embargo, para los valores de resistencia a la penetración (RP) del suelo sí se observaron diferencias significativas $(p<0,05)$ entre tratamientos, obteniendo el mayor promedio Lp+Tr ST con $2.322 \mathrm{kPa}$, respecto a estrategias que producen una escasa intervención en la estructura del suelo como NsF con $2.075 \mathrm{kPa}$ y NcF con $2.122 \mathrm{kPa}$. De la misma forma el tratamiento Lp+Tr ST presentó la mayor conductividad de aire del suelo con $1,05 \mathrm{~cm} \mathrm{~s}^{-1}$ respecto a los otros tratamientos.

De acuerdo al Cuadro 3, se observan poblaciones con distribuciones simétricas según los valores de kurtosis y skewness (Webster y Oliver, 2001). Además, se presentan coeficientes de variación bajos en el contenido volumétrico de agua $15,7 \%$ y resistencia a la penetración del suelo $12,7 \%$, lo que indica que la mayoría de los datos se concentran alrededor de su valor medio $y$, por tanto, la probabilidad de encontrar un valor cercano a éste aumenta (Webster y Oliver, 2001). La conductividad de aire es el parámetro que presentó el mayor coeficiente de variación $37,5 \%$.

Los parámetros físicos evaluados se ajustaron a un modelo exponencial (Cuadro 4), alcanzando valores de $R^{2}>0,55$ los que muestran un buen ajuste en los semi- 
Cuadro 2.Análisis estadístico de las propiedades físicas evaluadas en terreno. Letras distintas indican diferencias estadísticamente significativas entre los tratamientos $(\mathrm{p} \leq 0,05)$.

Table 2. Statistical analysis of the physical properties evaluated in the field. Different letters indicate statistically significant differences between treatments $(\mathrm{p} \leq 0.05)$.

\begin{tabular}{lccc}
\hline \multicolumn{1}{r}{ Tratamiento } & $\mathbf{K l}\left(\mathbf{c m ~ s}^{-1}\right)$ & $\boldsymbol{\theta c}($ Vol $\mathbf{~})$ & RP (kPa) \\
\hline NsF & $0,70 \pm 0,026 \mathrm{~b}$ & $19,73 \pm 0,56 \mathrm{a}$ & $2.075 \pm 39,51 \mathrm{~b}$ \\
$\mathbf{N c F}$ & $0,63 \pm 0,018 \mathrm{~b}$ & $20,06 \pm 0,55 \mathrm{a}$ & $2.122 \pm 55,40 \mathrm{~b}$ \\
$\mathbf{L p + T r}$ ST & $1,05 \pm 0,007 \mathrm{a}$ & $18,61 \pm 0,68 \mathrm{a}$ & $2.322 \pm 53,70 \mathrm{a}$ \\
$\mathbf{L p + T r}$ CL & $0,68 \pm 0,021 \mathrm{~b}$ & $19,61 \pm 0,56 \mathrm{a}$ & $2.177 \pm 43,11 \mathrm{ab}$ \\
Polifítica CL & $0,66 \pm 0,018 \mathrm{~b}$ & $18,74 \pm 0,66 \mathrm{a}$ & $2.155 \pm 55,50 \mathrm{ab}$ \\
\hline
\end{tabular}

Cuadro 3.Descripción estadística de las propiedades físicas del suelo evaluadas.

Table 3. Statistical description of the evaluated soil physical properties.

\begin{tabular}{lccc}
\hline & $\mathbf{K I}\left(\log \mathbf{~ c m ~ s}^{-1}\right)$ & $\boldsymbol{\theta}_{\mathbf{c}}(\%)$ & $\mathbf{R P}(\mathbf{k P a})$ \\
\hline $\mathbf{N}$ & 127 & 135 & 135 \\
Promedio & $-0,40$ & 19,49 & 2.175 \\
Media & $-0,34$ & 19,49 & 2.175 \\
Mediana & $-0,17$ & 19,2 & 2.119 \\
CV (\%) & 37,48 & 15,68 & 12,70 \\
Valor máximo & 0,095 & 27,2 & 2.901 \\
Valor mínimo & $-0,92$ & 12,4 & 1.630 \\
Skewness & $-0,04$ & 0,23 & 0,70 \\
Kurtosis & 0,68 & $-0,38$ & $-0,05$ \\
\hline
\end{tabular}

Cuadro 4. Parámetros geoestadísticos para definir la estructura de variación espacial de las propiedades del suelo.

Table 4. Geostatistical parameters to define the structure of spatial variability of soil properties.

\begin{tabular}{lccc}
\hline & $\mathbf{K I}\left(\mathbf{l o g} \mathbf{~ c m ~ s}^{-1}\right)$ & $\boldsymbol{\theta}_{\mathbf{c}}(\%)$ & $\mathbf{R P}(\mathbf{k P a})$ \\
\hline $\mathbf{N}$ & 127 & 135 & 135 \\
Promedio & $-0,40$ & 19,49 & 2.175 \\
Media & $-0,34$ & 19,49 & 2.175 \\
Mediana & $-0,17$ & 19,2 & 2.119 \\
CV (\%) & 37,48 & 15,68 & 12,70 \\
Valor máximo & 0,095 & 27,2 & 2.901 \\
Valor mínimo & $-0,92$ & 12,4 & 1.630 \\
Skewness & $-0,04$ & 0,23 & 0,70 \\
Kurtosis & 0,68 & $-0,38$ & $-0,05$ \\
\hline
\end{tabular}


variogramas. El rango (Ao) que obtuvieron la conductividad de aire y resistencia a la penetración del suelo fue de 201 metros, para el contenido volumétrico en cambio fue de 105 metros, todos ellos con valores entre 0,5 y 0,6 en la razón Co/C +Co indicando una moderada dependencia espacial según lo establecido por Cambardella et al. (1994).

A nivel general se observó una baja variabilidad en el contenido de agua en el suelo (Figura 2) con un pro- medio de $19,5 \%$ y valores que fluctúan entre $12,4 \%$ a $27,2 \%$. Por otro lado, se registró una mayor variabilidad espacial en la resistencia a la penetración del suelo (Figura 2), la cual osciló entre valores de $1.620 \mathrm{kPa}$ y $2.900 \mathrm{kPa}$, con un promedio a nivel general de 2.175 $\mathrm{kPa}$. Los sectores con mayor resistencia a la penetración correspondieron a las praderas con siembra tradicional y cero labranza, siendo la más destacada Lp+Tr ST, lo que además concuerda con los valores promedios
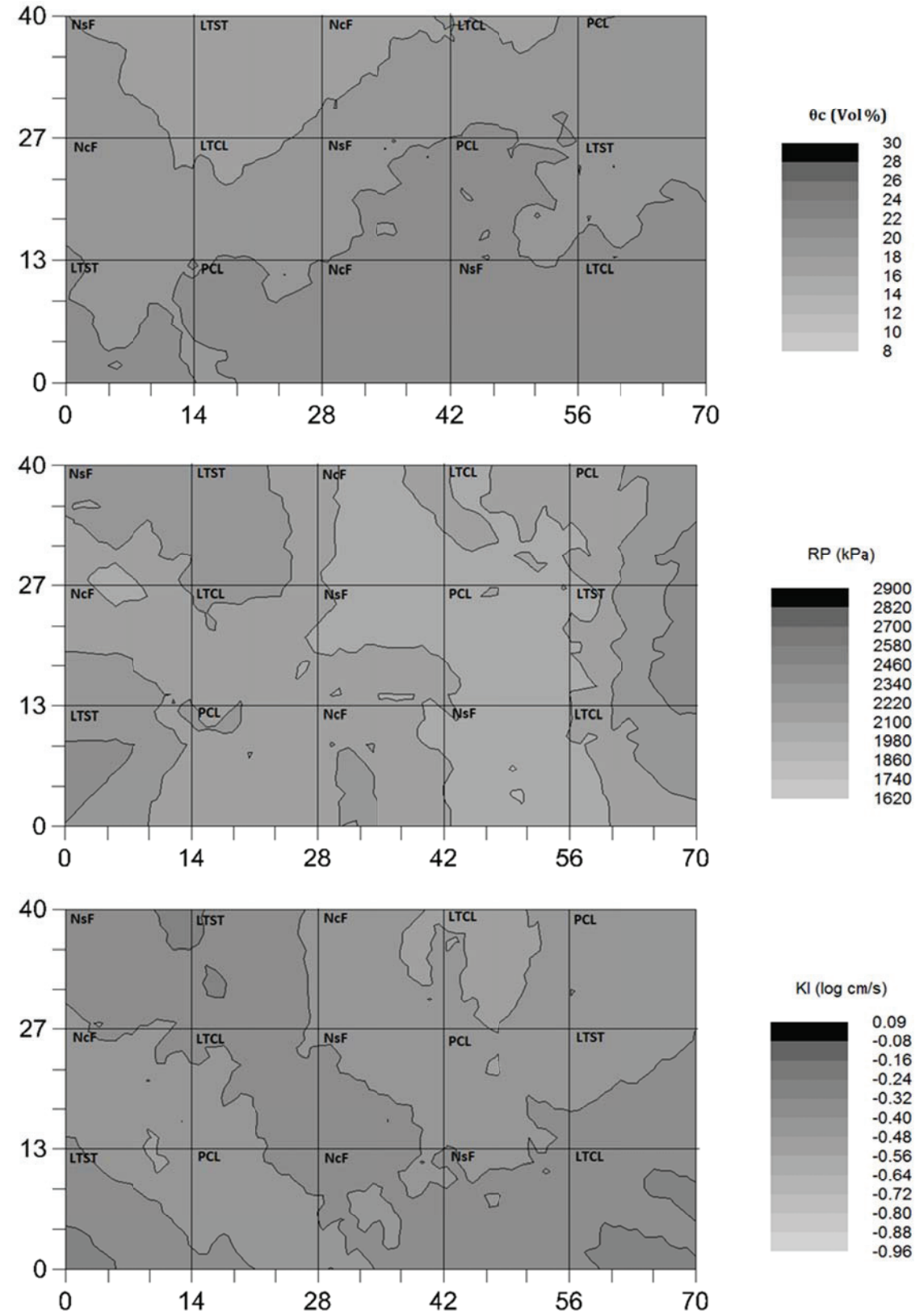

Figura 2. Variabilidad espacial del contenido volumétrico de agua $\left(\theta_{c}\right)$ resistencia a la penetración (RP) y conductividad de aire $(\mathrm{Kl})$ de el suelo para las distintas estrategias de mejoramiento de una pradera degradada.

Figure 2. Spatial variability of the volumetric water content $\left(\theta_{\mathrm{c}}\right)$, penetration resistance (RP) and air conductivity (Kl) of the soil for different grassland improvement strategies. 
de los tratamientos (Cuadro 2). Finalmente, la conductividad de aire en el suelo es la propiedad que presentó la mayor variabilidad espacial (Figura 2) con valores que estuvieron entre $-0,92 \log \mathrm{cm} \mathrm{s}^{-1}$ a 0,095 $\log \mathrm{cm} \mathrm{s}^{-1}$, áreas que presentaron la mayor capacidad de conducción de aire pertenecen al tratamiento $\mathrm{Lp}+\mathrm{Tr} \mathrm{ST}$, la que presentó el mayor promedio, de 1,05 $\mathrm{cm} \mathrm{s}^{-1}$ (Cuadro 2).

\section{Efecto del pastoreo ovino en propiedades físicas del suelo bajo diferentes estrategias de mejoramiento en una pradera degradada}

La Figura 3 mostró diferencias $(\mathrm{p}<0,05)$ en el contenido volumétrico de agua en el suelo por evento de pastoreo en los tratamientos NsF con un valor previo de 19,7\% y Lp+Tr ST de 18,6\% respecto a sus valores posteriores de $17,8 \%$ y $16,3 \%$, respectivamente. La disminución del contenido volumétrico de agua en el suelo de estos tratamientos fue de aproximadamente $1,9 \%$ y $2,3 \%$. Por otro lado, todos los tratamientos tuvieron valores similares de RP posterior a un evento de pastoreo (Figura 3), sin embargo, solo el suelo bajo la pradera Polifítica CL presenta diferencias estadísticamente significativas $(\mathrm{p}<0,05)$; previo al pastoreo RP fue de $2.155 \mathrm{kPa}$, aumentando a $2.322 \mathrm{kPa}$.

Todos los tratamientos presentan una disminución en su capacidad de conducir aire después de un evento de pastoreo (Figura 3), sin embargo, los tratamientos $\mathrm{NcF}$ con $0,63 \mathrm{~cm} \mathrm{~s}^{-1}, \mathrm{Lp}+\mathrm{Tr}$ ST con $1,05 \mathrm{~cm} \mathrm{~s}^{-1}$ y Polifítica CL con $0,66 \mathrm{~cm} \mathrm{~s}^{-1}$ son los que presentan pérdidas significativas $(\mathrm{p}<0,05)$ de $\mathrm{Kl}$, donde los valores posteriores son para NcF de $0,48 \mathrm{~cm} \mathrm{~s}^{-1} \mathrm{Lp}+\operatorname{Tr} \mathrm{ST} 0,71 \mathrm{~cm} \mathrm{~s}^{-1}$
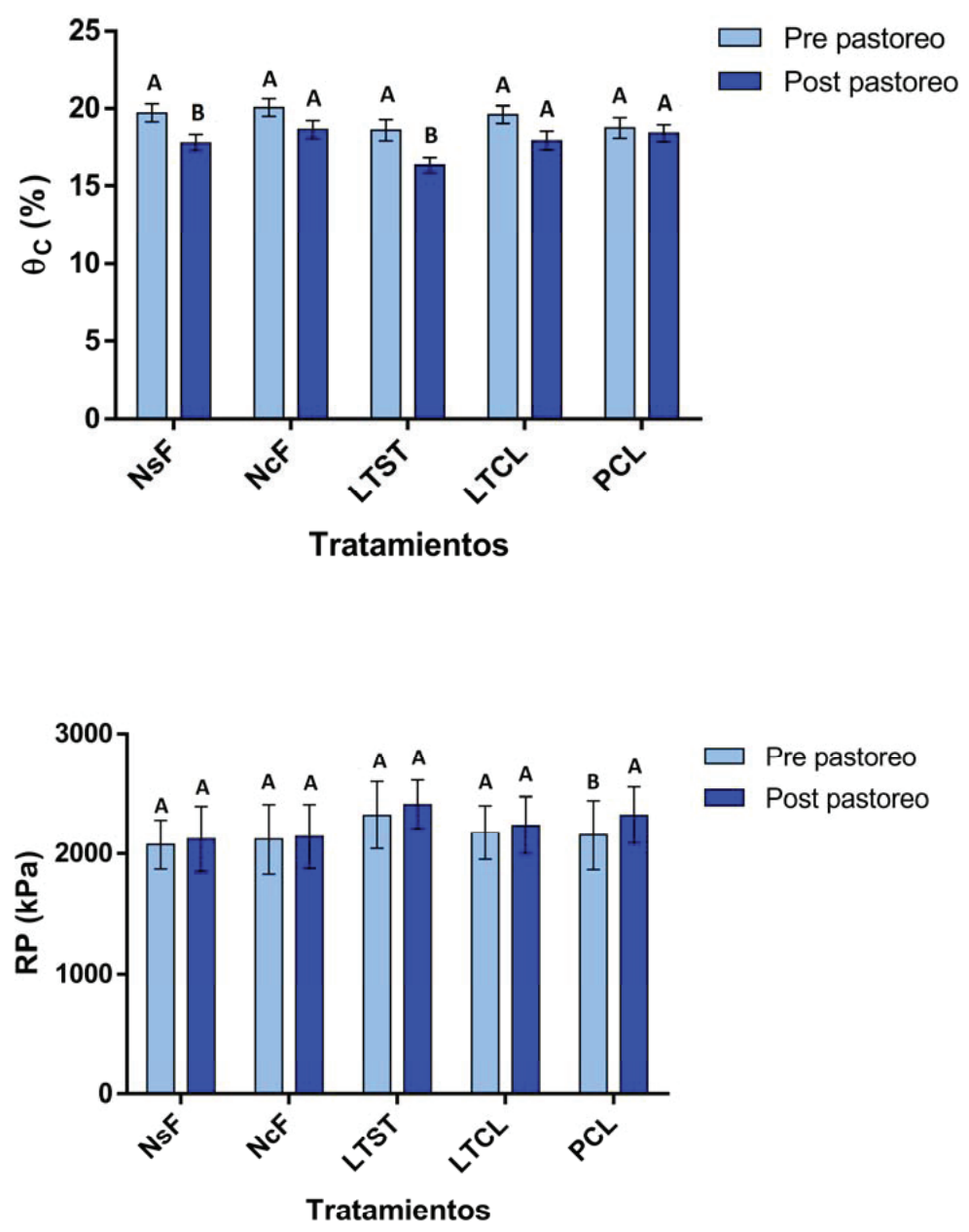

Figura 3. Contenido volumétrico de agua $\left(\theta_{c}\right)$ y Resistencia a penetración (RP) del suelo en función de estrategias de mejoramiento de una pradera degradada antes y después de un evento de pastoreo. Se presentan valores promedio \pm 1 error estándar. Letras distintas muestran diferencias $(\mathrm{p}<0,05)$ por efecto del pastoreo.

Figure 3. Volumetric water content $\left(\theta_{c}\right)$ and penetration resistance (RP) as function of the pasture improvement strategies before and after a grazing event. Mean values \pm 1 standard error is presented. Different letters show differences $(\mathrm{p}<0.05)$ due to grazing. 


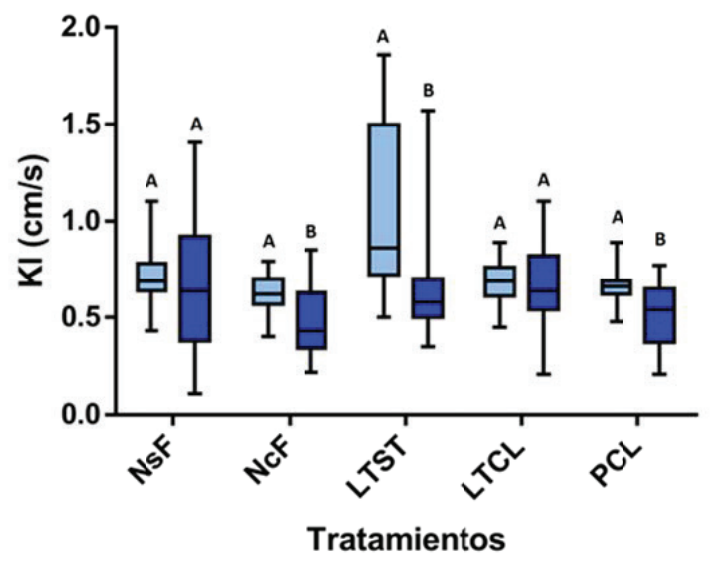

Figura 4. Conductividad de aire del suelo en función de estrategias de mejoramiento de una pradera degradada antes y después de un evento de pastoreo. Se presentan valores promedio \pm 1 error estándar. Letras distintas muestran diferencias $(\mathrm{p}<0,05)$ por efecto del pastoreo.

Figure 4. Air conductivity as function of the pasture improvement strategies before and after a grazing event. Mean values \pm 1 standard error is presented. Different letters show differences $(p<0.05)$ due to grazing.

y Polifítica $0,52 \mathrm{~cm} \mathrm{~s}^{-1}$. Esto significa una disminución de la conductividad de aire de 0,$15 ; 0,34$ y $0,14 \mathrm{~cm} \mathrm{~s}^{-1}$, respectivamente.

\section{DISCUSIÓN}

\section{Variabilidad espacial de algunas propiedades físicas del suelo bajo distintas estrategias de mejoramiento en una pradera degradada}

La variación espacial de una propiedad del suelo ocurre cuando una variable en un sitio depende de la distancia y/o la dirección (Jaramillo, 2008). La variabilidad de las propiedades del suelo, de un punto a otro del paisaje, tiene orígenes diversos, pudiendo provenir de las características inherentes a los procesos de formación de suelo predominante (Jaramillo, 2012), o bien de los factores de formación (Burrough, 1993). Es decir, la historia, el clima, la topografía, la actividad biológica y la acción humana, destacando de este último el efecto del manejo de la estructura del suelo (p. ej. Figura 3). Mientras algunos de los procesos y factores de formación afectan a pequeños volúmenes de suelo e introducen la heterogeneidad a distancias cortas, otros provocan dependencia espacial de más largo alcance (Ulloa et al., 2003).

El efecto de distintas estrategias de mejoramiento de una pradera sobre la variación espacial de propiedades físicas del suelo puede ser estudiada a través del uso de herramientas de evaluación in situ como en el presente estudio. Estas poseen una mayor capacidad para detectar variaciones temporales y espaciales, lo que permite p. ej., una mejor comprensión de los flujos preferenciales (Angulo-Jaramillo et al., 2000) o del efecto del cambio de uso de suelo sobre las propiedades físicas del suelo (Dörner et al., 2016). Sin embargo, al ser herramientas de evaluación in situ se debe considerar que sus resultados están condicionados a una fecha y lugar determinado, p.ej. lo que determina el contenido de agua del suelo (Figura 2, Cuadro 2) tal como lo discuten Dec et al. (2011). De acuerdo a los resultados presentados (Cuadro 2), en donde NsF es la situación inicial del suelo (pradera degradada sin fertilización), el contenido volumétrico de agua $\left(\theta_{c}\right)$ es bajo (cercano al punto de marchitez permanente, PMP) y no se muestran diferencias entre tratamientos tal como lo encontró Ordóñez (2015), para los mismos tratamientos. Por otro lado, los mapas de variación espacial de $\theta_{c}$ muestran variaciones tenues que en cierta medida indican una homogeneidad en la textura del suelo, ya que el contenido de agua del suelo en PMP está estrechamente relacionado con el contenido de arcilla del suelo (Hartge y Horn, 2009).

La resistencia a la penetración (RP) registrada en los tratamientos, luego de 30 meses de establecidas las estrategias de mejoramiento de praderas y considerando diversos pastoreos, excede el nivel crítico de $2.000 \mathrm{kPa}$ propuesto por Horn y Fleige (2009), lo que evidencia problemas de compactación de suelos cuando es determinado a un $\theta_{c}$ del suelo equivalente a capacidad de campo (que para este suelo corresponde a un contenido de agua de 23 \% Vol., de acuerdo a Dörner et al., 2013). Sin embargo, en este estudio los altos valores se debieron a que la RP aumenta significativamente con la reducción del contenido de agua del suelo (Dec et al., 2011), por lo tanto, en este caso la RP representa una mayor cohesión del suelo por la formación de meniscos de agua que incrementan la resistencia mecánica de los 
agregados (Hartge y Horn, 2009). En consecuencia, no se pueden asociar a un problema de compactación de suelo, tal como lo discutiera Dec et al. (2011) en la misma serie de suelo. De la misma forma no se presentaron valores críticos de Kl, lo que según Horn y Fleige (2009), ocurren cuando la conductividad de aire es menor a $5,5^{*} 10^{-4} \mathrm{~cm} \mathrm{~s}^{-1}$.

Los sectores con mayor resistencia a la penetración corresponden a praderas sembradas, siendo la que más afectada Lp+Tr ST (Figura 2). El efecto de haber sembrado y, establecido una mayor cantidad especies botánicas, no sólo produce un aumento de la producción de materia seca sobre la superficie del suelo, sino también una mayor abundancia de raíces y actividad biológica que aumentan la estabilidad estructural dentro y fuera de la rizósfera (Hinsinger et al., 2009). De acuerdo a esto, se ha indicado que las exudaciones de polisacáridos, realizadas por el género Rhizobium, tienen un efecto cementante, generando un incremento significativo en la agregación de la estructura y estabilidad estructural del suelo (North y Nobel 1997; Haynes y Beare, 1997; McCully, 1999; Moreno-Espíndola et al., 2007) lo que permite el aumento de la RP.

La conductividad de aire (Kl) del suelo es el parámetro que presentó la mayor variabilidad espacial (Figura 2). Esto, usualmente ese así debido a que es un parámetro físico que depende de múltiples factores como la época del año en que sea medido, en relación a las características de cada estación, temperatura, humedad, estructura, macroestructura, porosidad total, distribución de poros, continuidad y tortuosidad de poros y manejo del suelo lo que consecuentemente genera una mayor variabilidad (Dörner y Horn, 2006). Luego de 30 meses de establecidas las estrategias de mejoramiento, el tratamiento que presentó la mayor $\mathrm{Kl}$ respecto a los demás fue Lp+Tr ST. Estos resultados son contrarios a los presentados por Ordóñez (2015), quien registró los menores valores de capacidad y conductividad de aire del suelo en el mismo tratamiento para el mismo ensayo, pero medidos 5 y 18 meses después del establecimiento de las praderas. Para una correcta comparación e interpretación de los estudios hay que considerar que KI incluye aspectos dinámicos en el tiempo, debido a la constante formación y deformación de la estructura del suelo, que altera el comportamiento del sistema poroso en el tiempo. Por lo tanto, al existir mayor tiempo desde el establecimiento de las estrategias de mejoramiento de praderas en el presente estudio, los valores de Kl reflejan una recuperación de la estructura del suelo, debido principalmente a los procesos de mojado y secado (Dörner et al., 2009; Ivelic-Sáez et al., 2015), actividad biológica, crecimiento y muerte de raíces, además del efecto de los ácidos orgánicos presentes en el suelo debido a la actividad microbial (Horn y Smucker, 2005). En ese contexto, la alta resiliencia de estos suelos (Dörner et al., 2009; Dec et al., 2012), re- lacionada con el carbono orgánico que actúa como un elemento elástico, permite la recuperación volumétrica y funcional de los poros del suelo (Dörner et al., 2009). De esta forma, como resultado de la interacción de los procesos y factores anteriormente mencionados, se logra consolidar un desarrollo estructural en el tiempo que favorece particularmente a la macro estructura y el desarrollo de porosidad secundaria (Ellies et al., 1997). Esto facilita el movimiento de agua y aire en el suelo (Dörner y Dec, 2007) y permite que parámetros que definen "intensidad", que son los que evalúan la funcionalidad del suelo (p. ej. conductividad de aire) evolucionen positivamente en el tiempo, indicando el comienzo de una estabilización estructural del suelo en el mediano (Zúñiga et al., 2015) y largo plazo. Esto fue más significativo en tratamientos donde se establecieron especies botánicas como el L. perenne y T. repens que concentran sus raíces a nivel superficial del suelo (primeros $20 \mathrm{~cm}$ principalmente), por lo que logran un mayor efecto en la intensidad con que se produce la reestructuración del suelo en el tiempo. Por lo tanto, es posible relacionar estrategias con mayor resistencia a la penetración por efecto de mayor cantidad de raíces y por lo tanto un mayor consumo de agua, que permite el incremento del flujo de aire en el sistema poroso.

En relación a lo anteriormente mencionado, se debe tener en consideración la sequía registrada durante el verano del 2015 para el presente estudio, lo que consecuentemente se relacionaría con un evento severo de contracción del suelo, lo que se asocia a una mayor presencia de grietas y por lo tanto flujos preferenciales para el transporte de aire en el suelo.

El comportamiento espacial observado por el contenido volumétrico de agua y resistencia a la penetración del suelo coincide con resultados presentados por Dec et al. (2012) en donde la variación espacial de estos parámetros, mostraban que sectores con menor contenido de agua aumenta la resistencia mecánica. A su vez en el presente estudio se observa también que estos sectores presentan mayor conductividad de aire (especialmente en $\mathrm{NcF}$ y Lp+Tr ST), por lo que existe una mayor fracción de poros saturados con aire que permite un mayor flujo por convección (Dörner y Horn, 2006).

\section{Efecto del pastoreo ovino en propiedades físicas del suelo bajo diferentes estrategias de mejoramiento en una pradera degradada}

Los resultados presentados, muestran una disminución en el contenido volumétrico de agua del suelo $\left(\theta_{c}\right)$ después del pastoreo en todos los tratamientos, siendo significativo en NsF y Lp+Tr ST. En cuanto a Lp+Tr ST, además de tener un menor $\theta_{c}$ después de un evento de pastoreo, presenta la mayor conductividad de aire antes de ser pastoreada, indicativo de una alta continui- 
dad porosa, asociada a un mayor espacio poroso, por lo que consecuentemente, la presión ejercida sobre el suelo disminuye en una mayor proporción el espacio poroso, respecto a las demás estrategias, planteando una mayor sensibilidad de dicha estrategia a eventos de pastoreo.

A nivel general, se presenta un aumento de la RP del suelo por eventos de pastoreo, lo que coincide con los resultados presentados por Ordóñez (2015). Sin embargo, como es un estudio de campo no se puede concluir que este aumento sea consecuencia del efecto del pastoreo, ya que la reducción de $\theta_{c}$ implica también un incremento en la resistencia mecánica del suelo (Dec et al., 2011). Sin perjuicio de lo anterior, la reducción de la conductividad de aire después de un evento de pastoreo indica una deformación del suelo por efecto del pisoteo animal, presentando en Lp+Tr ST, NcF y Polifítica CL diferencias estadísticamente significativas $(\mathrm{p}<0,05)$. Por lo tanto, la reducción no significativa en la mayoría de los casos demuestra una estabilización de la estructura que le permite resistir los eventos de pastoreo sin afectar significativamente la capacidad de los poros del suelo luego de 30 meses de establecidas las distintas estrategias de mejoramiento de praderas. Sin embargo, respecto al funcionamiento de los poros se presenta un mayor efecto, lo que se responde con la mayor sensibilidad que estos presentan al provocarse disturbaciones en el suelo, en este caso por el pastoreo ovino.

\section{CONCLUSIONES}

Los parámetros seleccionados permiten registrar la variación espacial de las propiedades físicas del suelo. Se determinó una moderada dependencia espacial en todos los parámetros físicos evaluados en terreno, tal que sectores con mayor contenido volumétrico de agua $\left(\theta_{c}\right)$ en el suelo fueron los que presentaron menor resistencia a la penetración (RP) y conductividad de aire (Kl).

La conductividad de aire del suelo presentó la mayor variación espacial ya que depende del contenido de agua del suelo y también es un reflejo de la continuidad del sistema poroso.

Los 30 meses desde el establecimiento de las estrategias de mejoramiento de praderas han permitido una estabilización de la estructura del suelo que se refleja en el bajo impacto del pastoreo ovino sobre la capacidad física de los poros del suelo, sin embargo, el funcionamiento de los poros representado con la conductividad de aire, presenta una mayor sensibilidad por efecto del pastoreo.

\section{AGRADECIMIENTOS}

Se agradece el financiamiento del estudio a proyecto FONDECYT 1130795.

\section{REFERENCIAS}

Angulo-Jaramillo, R., Vandervaere, J., Roulier, S., Thony, L., Gaudet, P., Vauclin, P., 2000. Field measurement of soil surface hydraulic properties by disc and ring infiltrometers - a review and recent developments. Soil and Tillage Research 55, 1-29.

Balocchi, 0., 2002. Praderas y recursos forrajeros en la zona sur de Chile, in: Amtman, C., Mujica, F., Vera, B. (Eds.), Pequeña agricultura en la Región de Los Lagos, Chile. Universidad Austral de Chile, Valdivia, Chile. pp. 59-73.

Balocchi, O., López, I., 1994. Regeneración de praderas, in: Producción Animal. Latrille, L. (Ed.), Universidad Austral de Chile, Facultad de Ciencias Agrarias, Instituto de Producción Animal, Valdivia, Chile, pp. 263-293.

Balocchi, O., López, I., 1996. Especies pratenses nativas y naturalizadas del sur de Chile, in: Latrille, L. (Ed.), Producción Animal. Universidad Austral de Chile, Facultad de Ciencias Agrarias, Instituto de Producción Animal. Serie B - 20. Valdivia, Chile, pp. 65-81.

Beutler, A.N., Centurion, F.J., Silva, A.P., 2005. Soil resistance to penetration and least limiting water range for soybean yield in a Haplustox from Brasil. Brasilian Archives of Biology and Technology 48, 863-871.

Bravo, C., Cabrera, J., Carvallo, M., Chánchica, H., Ramírez, E., Gonzáles I., 2011. Variabilidad espacial de propiedades físicas y químicas del suelo y su relación con el rendimiento de frijol (Vigna unguiculata L.) en una parcela experimental de los llanos centrales venezolanos. Estudios en la Zona no Saturada del Suelo X, 79-84.

Burrough, P.A., 1993. Soil variability: a late 20 th century view. Soils and Fertilizers 56, 529-562.

Cambardella, C. A., Moorman, T. B., Novak, J. M., Parkin, T. B., Karlen, D. L., Turco, R. F., Konopka, A. E., 1994. Fieldscale variability of soil properties in Central Iowa soils. Soil Science Society of American Journal 58, 1501-1511.

Centro de Información de Recursos Naturales (CIREN), 2003. Estudio agrológico X Región. Descripción de Suelos, Materiales y Símbolos, Publicación CIREN N¹23, Santiago, Chile.

Cuevas, E., 1980. Manejo y utilización de praderas. Serie B-2. Universidad Austral de Chile, Facultad de Ciencias Agrarias, Instituto de Producción Animal. Valdivia, Chile.

Dec, D., Dörner, J., Balocchi, O., 2011. Temporal and spatial variability of structure dependent properties of a volcanic ash soil under pasture in southern Chile. Chilean Journal of Agricultural Research 71(2), 293-303.

Dec, D., Dörner., J., Balocchi, O., López, I., 2012. Temporal dynamics of hydraulic and mechanical properties of an Andosol under grazing. Soil and Tillage Research 125, 44-51.

Dörner, J., Horn, R., 2006. Anisotropy of pore functions in structured Stagnic Luvisols in the Weichselian moraine region in N Germany. Journal of Plant Nutrition and Soil Science 169, 213-220.

Dörner, J., Dec, D., 2007. La permeabilidad de aire y conductividad hidráulica saturada como herramientas para la caracterización funcional de los poros del suelo. Revista de la Ciencia del Suelo y Nutrición Vegetal 7(2), 1-13.

Dörner, J., Dec, D., Peng, X., Horn, R., 2009. Efecto del cambio de uso en la estabilidad de la estructura y la función de 
los poros de un Andisol (Typic Hapludand) del Sur de Chile. Revista de la Ciencia del Suelo y Nutrición Vegetal 9(3), 190-209.

Dörner, J., Dec, D., Peng, X., Horn, R., 2010. Effect of land use change on the dynamic behaviour of structural properties of an Andisol in southern Chile under saturated and unsaturated hydraulic conditions. Geoderma 159, 189197.

Dörner, J., Dec, D., Zúñiga, F., Sandoval, P., Horn, R., 2011. Effect of the land use change of a Typic Durudand on pore functions and their functional resilience after mechanical and hydraulic stress. Soil and Tillage Research 115-116, 71-79.

Dörner, J., Dec, D., Feest, F., Vásquez, F., Díaz, M., 2012. Dynamics of soil structure and pore functions of a volcanic ash soil under tillage. Soil and Tillage Research 125, 52-60.

Dörner, J., Dec, D., Zúñiga, F., Horn, R., López, I., Leiva, C., Cuevas, J., 2013. Soil Changes in the Physical Quality of an Andosol under Different Management Intensities in Southern Chile, in: Krümmelbein, J., R. Horn, M. Pagliai. (Eds.), Soil Degradation. Advances in Geoecology. Catena Verlag, Reiskirchen, Germany, pp. 262-281.

Dörner, J., Huertas, J., Cuevas, J.G., Leiva, C., Paulino, L., Arumí, J., 2015. Water content dynamics in a volcanic ash soil slope in southern Chile. Journal of Plant Nutrition and Soil Science 178(4), 693-702.

Dörner, J., Dec, D., Thiers, O., Paulino, L., Zúñiga, F., Valle, S., Martínez, O., Horn, R., 2016. Spatial and temporal variability of physical properties of Aquands under different land uses in southern Chile. Soil Use Management 32(3), 411-421.

Ellies, A., 1995. Efecto del manejo sobre las propiedades físicas de suelos trumaos y rojo arcillosos. Bosque 16(2), 101-109.

Ellies, A., Grez, R., Ramírez, C., 1997. La conductividad hidráulica en fase saturada como herramienta para el diagnóstico de la estructura del suelo. Agro Sur 25, 51-56.

Ellies, A., Ramírez, C., Macdonald, R., 1993. Variación en la resistencia del suelo por efecto de su uso. Turrialba 43(1), 77-82.

Eijkelkamp Agrisearch Equipment, 2014. 14.34.01 (PL-300). Instrument for field measurement of Air permeability, Soil moisture tension, Soil volumetric moisture tension. Users's Manual. Giesbeek, Netherlands. https://www. eijkelkamp.com/download.php?file=M1143401e_9123. pdf.

Hartge, R., Horn, R., 2009. Die physikalische Untersuchung von Bóden. Praxis Messmethoden Auswertung. 4. vollst. Überarbeitete Auflage. Schweizerbart Vorlage, Stuttgart.

Haynes, J., Beare, M., 1997. Influence of six crop species on aggregate stability and some labile organic matter fractions. Soil Biology and Biochemistry 29, 1647-1653.

Hinsinger, P., Gobran, G., Gregory, P., Wenzel, J., 2005. Rhizosphere geometry and heterogeneity arising from rootmediated physical and chemical processes. New Phytologist 168, 293-303.

Horn, R., Baumgartl, T., 2001. Dynamic properties of soils, in: Warrick, A. (Ed.), Soil Physics Companion CRC press, Florida, pp. 17-48.

Horn, R., Smucker, A., 2005. Structure formation and its consequences for gas and water transport in unsaturated arable and forest soils. Soil and Tillage Research 82, 5-14.

Horn, R., Kutilek. M., 2009. The intensity-capacity conceptHow far is it possible to predict intensity values with capacity parameters. Soil and Tillage Research 103, 1-3.

Horn, R., Fleige, H., 2009. Risk assessment of subsoil compaction for arable soils in Northwest Germany at farm scale. Soil and Tillage Research 102, 201-208.

Huber, A., 1970. Diez años de observaciones climatológicas en la estación Teja-Valdivia (Chile) 1960-1969. Valdivia, Chile, Universidad Austral de Chile. 60 p.

Instituto Nacional de Estadísticas (INE), 2007. Censo agropecuario y forestal 2007. Resultados por comuna http:// www.ine.cl/canales/chile_estadistico/censos_agropecuarios/censo_agropecuario_07_comunas.php.

Ivelic-Sáez, J., Zúñiga, F., Valle, S., López, I., Dec, D., Dörner, J., 2015. Functional resistance and resilience of the pore system of an Andisol exposed to different strategies of pasture improvement under sheep grazing. Journal of Soil Science and Plant Nutrition 15, 663-679.

Jaramillo, D., 2008. Variabilidad espacial de las propiedades químicas del epipedón de un Andisol hidromórfico del oriente antioqueño (Colombia). Revista Facultad Nacional de Agronomía 61(2), 4588-4599.

Jaramillo, D., 2012. Variabilidad espacial del suelo bases para su estudio. Revista Facultad Nacional de Agronomía 1(1), 73-87.

McCully, M., 1999. Roots in soil: unearthing the complexities of roots and their rhizospheres. Annual Review of Plant Physiology and Plant Molecular Biology 50, 695-718.

Moreno-Espíndola, I., Rivera-Becerril., F., Ferrara-Guerrero, M., De León-González, F., 2007. Role of root-hairs and hyphae in adhesion of sand particles. Soil Biology and Biochemistry 39, 2520-2526.

Nielsen, D.R., Wendroth, O., 2003. Spatial and temporal statistics: sampling field soils and their vegetation. Catena Verlag, Reiskirchen.

North, G., Nobel, P., 1997. Drought-induced changes in soil contact and hydraulic conductivity for roots of opuntia ficus-indica with and without rhizosheaths. Plant Soil 191, 249-258.

Ordóñez, I., 2015. Evaluación de estrategias de mejoramiento de praderas y su efecto sobre el funcionamiento de los poros de un suelo derivado de cenizas volcánicas bajo pastoreo ovino. Tesis Magíster, Universidad Austral de Chile. 97 p.

Siebald, E., 2001. Mejoramiento de Praderas Naturalizadas, in: Opazo, L., Torres, A., Siebald, E. (Eds.), Seminario Praderas, Hacia un nuevo estilo productivo, Serie actas $\mathrm{N}^{\circ}$ 9, Centro Regional de Investigaciones Remehue, Osorno, Chile pp. 2-9. http://biblioteca.inia.cl/medios/biblioteca/serieactas/NR26893.pdf

Torres, A., 2006. Mejoramiento de Praderas Naturalizadas. Instituto de Investigación agropecuarias (INIA), Centro de Regional de Investigación Remehue Boletín INIA N ${ }^{\circ}$ 148, pp. 25-34. http://www2.inia.cl/medios/biblioteca/boletines/NR33836.pdf

Ulloa, M., Vidal, E., Ingaramo, O., Morales, L., Paz, A., 2003. Aplicación de la geoestadística al estudio de las propiedades del suelo. Universidad Nacional del Nordeste, Comunicaciones Científicas y Tecnológicas, Argentina, 
pp. 1-4. http://www.unne.edu.ar/unnevieja/Web/cyt/ cyt/2003/comunicaciones/05-Agrarias/A-031.pdf

Unger, P.W., 1996. Soil bulk density, penetration resistance, and hydraulic conductivity under controlled traffic conditions. Soil and Tillage Research 37, 67-75.

Vásquez, N., Salazar, F., Dörner, J., 2012. Variabilidad temporal de las propiedades físico-mecánicas de un suelo derivado de cenizas volcánicas bajo labranza convencional.
Agro Sur 40(3), 1-13.

Webster, R., Oliver, M.A., 2001. Geostatistics for environmental Scientists. John Wiley, Chichester, England.

Zúñiga, F., Ivelic-Sáez, J., López, I., Huygens, D., Dörner, J., 2015. Temporal dynamics of the physical quality of an Andisol under a grazing system subjected to different pasture improvement strategies. Soil and Tillage Research 145, 233-241. 